\title{
DIVERGENCE OF THE FOURIER SERIES OF CONTINUOUS FUNCTIONS WITH A RESTRICTION ON THE FRACTALITY OF THEIR GRAPHS ${ }^{1}$
}

\author{
Maxim L. Gridnev \\ Krasovskii Institute of Mathematics and Mechanics, \\ Ural Branch of the Russian Academy of Sciences, \\ Ekaterinburg, Russia, \\ coraxcoraxg@gmail.com
}

\begin{abstract}
We consider certain classes of functions with a restriction on the fractality of their graphs. Modifying Lebesgue's example, we construct continuous functions from these classes whose Fourier series diverge at one point, i.e. the Fourier series of continuous functions from this classes do not converge everywhere.
\end{abstract}

Key words: Trigonometric Fourier series, Fractality, Divergence at one point, Continuous functions.

Let $f$ be a $2 \pi$-periodic integrable function, and let

$$
\frac{a_{0}}{2}+\sum_{k=1}^{\infty}\left(a_{k} \cos k x+b_{k} \sin k x\right)
$$

where

$$
a_{k}=\frac{1}{\pi} \int_{-\pi}^{\pi} f(t) \cos k t d t, \quad b_{k}=\frac{1}{\pi} \int_{-\pi}^{\pi} f(t) \sin k t d t,
$$

be the trigonometric Fourier series of the function $f$. Denote by $S_{n}(f, x)$ the $n$th partial sum of (1). It is known (see [1, Ch. 1, Sect. 39]) that if $f$ has bounded variation on the period $(f \in B V)$, then its Fourier series converges everywhere on $\mathbb{R}$, and if, in addition, $f$ is continuous on $\mathbb{R}$, then the Fourier series converges to $f$ uniformly on $\mathbb{R}$. Salem [2] (see also [1, Ch. 4, Sect. 5]) considered the classes $B V_{p}$ of functions of bounded $p$-variation and proved that if $f \in B V_{p}$, then the Fourier series of $f$ also converges everywhere on $\mathbb{R}$. (Further generalizations of these results see in [3]).

The author [4] studied relations between the classes $B V_{p}$ and classes of continuous functions with a restriction on the fractality of their graphs.

Definition 1. Let $f: \mathbb{R} \rightarrow \mathbb{R}$ be a bounded $2 \pi$-periodic function. By the modulus of fractality of the function $f$, we call the function $\nu(f, \varepsilon)$ which, for all $\varepsilon>0$, gives the minimal number of closed squares with sides of length $\varepsilon$ parallel to the coordinate axes that cover the graph of the function $f$ on $[-\pi, \pi]$.

Definition 2. Let $\mu:(0,+\infty) \rightarrow \mathbb{R}$ be a nonincreasing continuous function such that $\lim _{\varepsilon \rightarrow 0} \mu(\varepsilon)=+\infty$. We define the functional class

$$
F^{\mu}:=\left\{f \in C_{2 \pi}: \nu(f, \varepsilon)=O(\mu(\varepsilon))\right\} .
$$

In the case $\mu(\varepsilon)=1 / \varepsilon^{\alpha}$, where $1 \leqslant \alpha \leqslant 2$, we will write $F_{\alpha}$ instead of $F^{1 / \varepsilon^{\alpha}}$.

\footnotetext{
${ }^{1}$ This work was supported by the Russian Science Foundation (project no. 14-11-00702).
} 
The following statements were proved in [4]:

$$
\begin{gathered}
B V=B V_{1}=F_{1} \quad[4, \text { Theorem 1]; } \\
B V_{p} \subset F_{2-1 / p}, \quad p>1 \quad[4, \text { Theorem 2]. }
\end{gathered}
$$

The latter is unimprovable; i.e., $B V_{p+\varepsilon} \nsubseteq F_{2-1 / p}$ for all $\varepsilon>0$.

In the present paper, we study the pointwise behavior of the Fourier series of continuous functions from $F^{\mu}$.

Theorem 1. Let $\mu:(0,+\infty) \rightarrow \mathbb{R}$ be a nonincreasing continuous function, let $\varepsilon \mu(\varepsilon)$ be a nonincreasing function, and let

$$
\lim _{\varepsilon \rightarrow+0} \varepsilon \mu(\varepsilon)=+\infty
$$

Then there exists a continuous function $F^{\mu}$ whose Fourier series does not converge everywhere.

P r o of of Theorem 1. We will require that

$$
\varepsilon^{-1}<\mu(\pi \varepsilon) \leqslant 2 \varepsilon^{-\frac{3}{2}}, \quad \varepsilon \in(0,1] .
$$

By (3), the former inequality holds on an interval $(0, \delta)$ and, changing the function $\mu$ on the interval $\left(\frac{\delta}{2}, 1\right)$, we will obtain the same class $F^{\mu}$. The latter inequality can only reduce the class $F^{\mu}$. Thus, if we find a required function in the narrower class, it will belong to the wider class immediately.

To obtain a function $f \in F^{\mu}$ with divergent Fourier series, we modify Lebesgue's example from [1, Ch. 1, Sect. 46]. We start with defining an increasing sequence of natural numbers $\left\{a_{k}\right\}$ as follows. Let $a_{0}=1$. Suppose that the first $k$ elements $a_{0}, a_{1}, \ldots, a_{k-1}$ have been already defined.

From inequalities (4), it follows that

$$
\frac{a_{k-1}^{2}}{a_{k-1}}<3 \mu\left(\frac{\pi}{a_{k-1}}\right)
$$

and, for $b \geqslant\left(6 a_{k-1}\right)^{2}$,

$$
\frac{b^{2}}{a_{k-1}} \geqslant 3 \mu\left(\frac{\pi}{b}\right)
$$

Then, by continuity, there exists the smallest number $a$ such that

$$
\frac{a^{2}}{a_{k-1}}=3 \mu\left(\frac{\pi}{a}\right)
$$

As $a_{k}$, we take the largest integer such that $a_{k} \leqslant a$ and the fraction $a_{k} / a_{k-1}$ is integer. It is not hard to understand that $a_{k}$ belongs to $\left[a-a_{k-1}, a\right]$, and, in view of the inequalities

$$
\frac{a_{k}}{a_{k-1}} \geqslant \frac{a-a_{k-1}}{a_{k-1}}=3 \mu\left(\frac{\pi}{a}\right) \frac{1}{a}-1 \geqslant 2,
$$

we conclude that $a_{k}>a_{k-1}$.

The definition of $a_{k}$ implies the inequality

$$
\frac{1}{\varepsilon^{2} a_{k-1}} \leqslant 3 \mu(\pi \varepsilon), \quad \varepsilon \in\left[\frac{1}{a_{k}}, 1\right] .
$$

The definition of $a_{k}$, inequalities (5), and condition (3) imply that

$$
\frac{a_{k}}{a_{k-1}} \rightarrow+\infty, \quad k \rightarrow+\infty .
$$


Consider the half-open intervals

$$
I_{k}=\left(\frac{\pi}{a_{k}}, \frac{\pi}{a_{k-1}}\right], \quad k \in \mathbb{N} .
$$

Let $\left\{k_{i}\right\}_{i=0}^{\infty}, k_{0}=1$, be an increasing sequence, on which, in what follows, two additional conditions will be imposed. Let

$$
c_{k}=\left\{\begin{array}{cl}
\sqrt{\frac{1}{\ln a_{k} / a_{k-1}},}, & k \in\left\{k_{i}\right\}_{i=0}^{\infty} ; \\
0, & k \notin\left\{k_{i}\right\}_{i=0}^{\infty} .
\end{array}\right.
$$

Finally, we define the function $f$ on the interval $[-\pi, \pi]$ :

$$
\begin{gathered}
f(x)=c_{k} \sin a_{k} x, \quad x \in I_{k}, \\
f(0)=0, \\
f(-x)=f(x) .
\end{gathered}
$$

We extend the function $f$ to $\mathbb{R}$ periodically. The resulting function is continuous on each $I_{k}$ and, since $a_{k} / a_{k-1}$ is integer, is continuous and vanishes at the points $\pm \pi / a_{k}$. Thus, the function $f$ is continuous on $[-\pi, \pi]$.

Since $f$ has only a finite number of maxima and minima on $[\delta, \pi], \delta>0$, it has bounded variation on this interval (and on $[-\pi,-\delta]$ as well). Thus, its Fourier series converges at every $x \in[-\pi, \pi] \backslash\{0\}$.

Consider now the sequence of partial sums of the Fourier series of $f$ at the point $x=0$. As is known [1, Ch. 1, Sect. 32, formula (32.5)], for the function $f$, we have

$$
S_{k}(x, f)=\frac{1}{\pi} \int_{-\pi}^{\pi} f(x+t) \frac{\sin k t}{t} d t+o(1)
$$

hence, for $x=0$,

$$
S_{k}(0, f)=\frac{1}{\pi} \int_{-\pi}^{\pi} f(t) \frac{\sin k t}{t} d t+o(1) .
$$

The function $f$ is even; therefore,

$$
S_{k}(0, f)=\frac{2}{\pi} \int_{0}^{\pi} f(t) \frac{\sin k t}{t} d t+o(1)
$$

Let us show that, after an appropriate choice of $\left\{k_{i}\right\}$,

$$
J_{i}=\int_{0}^{\pi} f(t) \frac{\sin a_{k_{i}} t}{t} d t \rightarrow+\infty, \quad i \rightarrow+\infty .
$$

Then $S_{a_{k_{i}}}(0, f) \rightarrow+\infty$ as $i \rightarrow+\infty$, i.e., the Fourier series of $f$ diverges at $x=0$.

To estimate $J_{i}$, we divide it into three terms:

$$
J_{i}=\int_{0}^{\pi / a_{k_{i}}} f(t) \frac{\sin a_{k_{i}} t}{t} d t+\int_{\pi / a_{k_{i}}}^{\pi / a_{k_{i}-1}} f(t) \frac{\sin a_{k_{i}} t}{t} d t+\int_{\pi / a_{k_{i}-1}}^{\pi} f(t) \frac{\sin a_{k_{i}} t}{t} d t=J_{i}^{\prime}+J_{i}^{\prime \prime}+J_{i}^{\prime \prime \prime}
$$

We have

$$
\left|\frac{\sin a_{k_{i}} t}{t}\right| \leqslant a_{k_{i}} .
$$

Hence,

$$
\left|J_{i}^{\prime}\right| \leqslant \max _{0 \leqslant t \leqslant \pi / a_{k_{i}}}|f(t)| a_{k_{i}} \frac{\pi}{a_{k_{i}}}=\pi c_{k_{i+1}}=o(1) .
$$


Suppose that $k_{1}, \ldots, k_{i-1}$ have been already defined. Then the function $f(t) / t$ is defined, bounded, and continuous on $\left(\pi / a_{k_{i}-1}, \pi\right]$. Extending this function by zero to $[-\pi, \pi]$ and assuming that $k_{i}$ are large enough (this is the first of two conditions on $k_{i}$ ), we can make the Fourier coefficient $a_{k_{i}}$ of the obtained function small enough; more precisely,

$$
\left|J_{i}^{\prime \prime \prime}\right|=\left|\int_{\pi / a_{k_{i}-1}}^{\pi} \frac{f(t)}{t} \sin a_{k_{i}} t d t\right| \leqslant \frac{1}{i} .
$$

It remains to estimate $J_{i}^{\prime \prime}$. We have

$$
\begin{gathered}
J_{i}^{\prime \prime}=\int_{\pi / a_{k_{i}}}^{\pi / a_{k_{i}-1}} c_{k_{i}} \sin a_{k_{i}} t \frac{\sin a_{k_{i}} t}{t} d t=\frac{c_{k_{i}}}{2} \int_{\pi / a_{k_{i}}}^{\pi / a_{k_{i}-1}} \frac{1-\cos 2 a_{k_{i}} t}{t} d t \\
=\frac{c_{k_{i}}}{2} \ln \frac{a_{k_{i}}}{a_{k_{i}-1}}-\frac{c_{k_{i}}}{2} \int_{\pi / a_{k_{i}}}^{\pi / a_{k_{i}-1}} \frac{\cos 2 a_{k_{i}} t}{t} d t .
\end{gathered}
$$

According to the second mean value theorem, taking into account that the function $1 / t$ is positive and monotone, we find that

$$
\left|\int_{\pi / a_{k_{i}}}^{\pi / a_{k_{i}-1}} \frac{\cos 2 a_{k_{i}} t}{t} d t\right| \leqslant \frac{a_{k_{i}}}{\pi}\left|\int_{\pi / a_{k_{i}}}^{\xi} \cos 2 a_{k_{i}} t d t\right| \leqslant \frac{a_{k_{i}}}{\pi} \frac{2}{2 a_{k_{i}}}=\frac{1}{\pi} .
$$

Thus,

$$
J_{i}^{\prime \prime}=\frac{c_{k_{i}}}{2} \ln \frac{a_{k_{i}}}{a_{k_{i}-1}}+o(1)
$$

Combining (8), (9), (10), and (11), and taking into account (7), we conclude that

$$
J_{i}=\frac{c_{k_{i}}}{2} \ln \frac{a_{k_{i}}}{a_{k_{i}-1}}+o(1)=\frac{1}{2} \sqrt{\ln \frac{a_{k_{i}}}{a_{k_{i}-1}}}+o(1) \rightarrow+\infty .
$$

Let us now estimate the modulus of fractality $\nu(f, \varepsilon)$. Denote by $\nu(f, \varepsilon)_{[a, b]}$ the minimal number of squares with sides of length $\varepsilon$ parallel to the coordinate axes that cover the graph of the function $f$ on $[a, b]$.

If $k_{1}, \ldots, k_{i-1}$ have been already defined, then the function $f$ is defined on the interval $\left[\pi / a_{k_{i-1}}, \pi\right]$ and has bounded variation; hence, by (2),

$$
\nu(f, \varepsilon)_{\left[\pi / a_{k_{i-1}}, \pi\right]}=O\left(\frac{1}{\varepsilon}\right) .
$$

Condition (3) allows us to take $k_{i}$ such that, for $\pi \varepsilon \in\left(0, \pi / a_{k_{i}}\right]$,

$$
\nu(f, \pi \varepsilon)_{\left[\pi / a_{k_{i-1}}, \pi\right]} \leqslant \mu(\pi \varepsilon) .
$$

This is the second condition on $k_{i}$.

Let $0<\varepsilon \leqslant 1$. Then there exists $i \in \mathbb{N}$ such that $\varepsilon \in\left[1 / a_{k_{i+1}}, 1 / a_{k_{i}}\right]$. Let us prove the inequality $\nu(f, \pi \varepsilon) \leqslant C \mu(\pi \varepsilon)$ with some constant $C$. It follows from what is proved above that the required inequality holds for the covering of the graph on $\left[\pi / a_{k_{i-1}}, \pi\right]$. The inequality also holds for the intervals $\left[\pi / a_{k_{i+1}-1}, \pi / a_{k_{i}}\right]$ and $\left[\pi / a_{k_{i}-1}, \pi / a_{k_{i-1}}\right]$ where $f$ is identically zero; hence,

$$
\nu(f, \pi \varepsilon)_{\left[\pi / a_{k_{i+1}-1}, \pi / a_{k_{i}}\right]}+\nu(f, \pi \varepsilon)_{\left[\pi / a_{k_{i}-1}, \pi / a_{k_{i-1}}\right]} \leqslant \frac{\pi}{\varepsilon} .
$$


Covering the whole rectangle $\left[0, \pi / a_{k_{i+1}-1}\right] \times\left[-c_{k_{i}}, c_{k_{i}}\right]$ and using (6), we can obtain the estimate

$$
\nu(f, \pi \varepsilon)_{\left[0, \pi / a_{k_{i+1}-1}\right]} \leqslant\left\lceil\frac{\pi}{a_{k_{i+1}-1} \pi \varepsilon}\right\rceil\left\lceil\frac{2 c_{k_{i}}}{\pi \varepsilon}\right\rceil \leqslant \frac{8}{a_{k_{i+1}-1} \pi \varepsilon^{2}} \leqslant \frac{24}{\pi} \mu(\pi \varepsilon) ;
$$

here and in what follows, $\lceil x\rceil$ stands for the rounding of $x$ upward.

It remains to cover the graph on the interval $\left[\pi / a_{k_{i}}, \pi / a_{k_{i}-1}\right]$ where $f(x)=c_{k_{i}} \sin a_{k_{i}} x$. We can divide this interval into $N_{i}=2 a_{k_{i}} / a_{k_{i}-1}-2$ intervals of monotonicity of $f$ : $\left[\pi / a_{k_{i}}+\pi(n-1) / 2 a_{k_{i}}, \pi / a_{k_{i}}+\pi n / 2 a_{k_{i}}\right], \quad n=1, \ldots, N_{i}$. Let us show that, to cover the graph of $f$ on each of these intervals, we need at most $8 / \pi \varepsilon$ squares. Using the definition of the length of a curve, we can show that the length of the graph of $f$ on these intervals is at most $\pi / 2 a_{k_{i}}+2 c_{k_{i}}$. Squares with sides of length $\pi \varepsilon$ can cover the graph of a monotone function of length at least $\pi \varepsilon$. Hence,

$$
\nu(f, \pi \varepsilon)_{\left[\pi / a_{k_{i}}+\pi(n-1) / 2 a_{k_{i}}, \pi / a_{k_{i}}+\pi n / 2 a_{k_{i}}\right]} \leqslant\left\lceil\left(\frac{\pi}{2 a_{k_{i}}}+2 c_{k_{i}}\right) \frac{1}{\pi \varepsilon}\right\rceil \leqslant \frac{8}{\pi \varepsilon} .
$$

From (6) and the monotonicity of $\varepsilon \mu(\varepsilon)$, we obtain

$$
\nu(f, \pi \varepsilon)_{\left[\pi / a_{k_{i}}, \pi / a_{k_{i}-1}\right]} \leqslant \frac{4 a_{k_{i}}}{\pi \varepsilon a_{k_{i}-1}} \leqslant \frac{12 \mu\left(\frac{\pi}{a_{k_{i}}}\right)}{\pi \varepsilon a_{k_{i}}}=\frac{12 \mu\left(\frac{\pi}{a_{k_{i}}}\right) \frac{\pi}{a_{k_{i}}} \mu(\pi \varepsilon)}{\pi^{2} \varepsilon \mu(\pi \varepsilon)} \leqslant \frac{12}{\pi} \mu(\pi \varepsilon) .
$$

Finally, by (12), (13), (14), and (15), we obtain the following estimate for the modulus of fractality of $f$ :

$$
\begin{gathered}
\nu(f, \pi \varepsilon) \leqslant 2 \nu(f, \pi \varepsilon)_{[0, \pi]} \leqslant 2\left(\nu(f, \pi \varepsilon)_{\left[0, \pi / a_{k_{i+1}-1}\right]}+\nu(f, \pi \varepsilon)_{\left[\pi / a_{k_{i+1}-1}, \pi / a_{k_{i}}\right]}\right] \\
\left.+\nu(f, \pi \varepsilon)_{\left[\pi / a_{k_{i}}, \pi / a_{k_{i}-1}\right]}+\nu(f, \pi \varepsilon)_{\left[\pi / a_{k_{i}-1}, \pi / a_{k_{i-1}}\right]}+\nu(f, \pi \varepsilon)_{\left[\pi / a_{k_{i-1}}, \pi\right]}\right) \\
\leqslant 2\left(\frac{24}{\pi} \mu(\pi \varepsilon)+\frac{\pi}{\varepsilon}+\frac{12}{\pi} \mu(\pi \varepsilon)+\mu(\pi \varepsilon)\right)=O(\mu(\pi \varepsilon))
\end{gathered}
$$

i.e., $f \in F^{\mu}$.

The theorem is proved.

\section{REFERENCES}

1. Bari N.K. Trigonometric series. Moscow: Fizmatgiz, 1961. 936 p. [in Russian].

2. Salem R. Essais sur les séries trigonométriques. Actual. Sci. et Industr. Vol. 862, 1940.

3. Waterman D. On converges of Fourier series of functions of generalized bounded variation // Studia Mathematica, 1972. Vol. 44. P. 107-117.

4. Gridnev M. L. About classes of functions with a restriction on the fractality of their graphs // CEURWS Proceedings, 2017. Vol.1894: Proceedings of the 48th Intern. Youth School-Conf.: Modern Problems in Mathematics and its Applications, Ekaterinburg, February 5-11, 2017. P. 167-173. http://ceurws.org/Vol-1894/appr5.pdf [in Russian]. 